\title{
Room Temperature Single-Photon Source: Single-Dye Molecule Fluorescence in Liquid Crystal Host
}

\author{
Svetlana G. Lukishova, Ansgar W. Schmid, Andrew J. McNamara, Robert W. Boyd, and Carlos R. Stroud, Jr.
}

\begin{abstract}
We report on new approaches toward an implementation of an efficient, room temperature, deterministically polarized, single-photon source (SPS) on demand-a key hardware element for quantum information and quantum communication. Operation of a room temperature SPS is demonstrated via photon antibunching in the fluorescence from single terrylene-dye molecules embedded in a cholesteric liquid crystal host. Using oxygen-depleted liquid crystal hosts, dye-bleaching was avoided over the course of more than $1 \mathrm{~h}$ of continuous 532-nm excitation. Liquid crystal hosts (including liquid crystal oligomers/polymers) permit further increase of the efficiency of the source: 1) by aligning the dye molecules along a direction preferable for maximum excitation efficiency; 2) by tuning a one-dimensional (1-D) photonic-band-gap microcavity of planar-aligned cholesteric (chiral nematic) liquid crystal layer to the dye fluorescence band.
\end{abstract}

Index Terms-Cholesteric liquid crystals, photoluminescence, single-photon source (SPS).

\section{INTRODUCTION}

Q UANTUM information in the form of quantum communications and quantum computing is an exceedingly active field today [1]-[6]. Numerous theoretical concepts promise powerful quantum-mechanics-based tools that, to date, wait for realization pending the arrival of reliable hardware. A single-photon source (SPS) [7], [8] that efficiently produces photons with antibunching characteristics [9], [10] is one such pivotal hardware element for quantum information technology. Using a SPS, secure quantum communication will prevent any potential eavesdropper from intercepting a message without the receiver's noticing [4], [11]. In another implementation, a SPS becomes the key hardware element for quantum computers with linear optical elements and photodetectors [12]-[14], [53]-[55]. Again, its practical realization is held back in part by difficulties in developing robust sources of antibunched photons on demand. In spite of several solutions for SPSs presented in the literature, significant drawbacks remain. They are the reason for

Manuscript received March 13, 2003; revised August 8, 2003. This work was supported in part by the U.S. Army Research Office under Award DAAD19-02-1-0285, the U.S. Department of Energy Office of Inertial Confinement Fusion under Cooperative Agreement DE-FC03-92SF19460, the University of Rochester, and the New York State Energy Research and Development Authority. The support of DOE does not constitute an endorsement by DOE of the views expressed in this paper.

S. G. Lukishova, A. J. McNamara, R. W. Boyd, and C. R. Stroud, Jr. are with the Institute of Optics, University of Rochester, Rochester, NY 14627 USA (e-mail: lukishov@optics.rochester.edu).

A. W. Schmid is with the Laboratory for Laser Energetics, University of Rochester, Rochester, NY 14623 USA (e-mail: asch@1le.rochester.edu).

Digital Object Identifier 10.1109/JSTQE.2003.820944 current quantum communication systems being baud-rate bottlenecked so that photon numbers from ordinary photon sources may be attenuated to the single-photon level ( $\sim 0.1$ photon per pulse on average) [4], [11]. An efficient (with an order of magnitude higher photon number per pulse) and reliable light source that delivers a train of pulses containing one and one photon only is a very timely challenge. To meet this challenge, several issues need addressing, from achieving full control of the quantum properties of the source to easy handling and integrability of these properties in a practical quantum computer and/or communication setup. In addition, in quantum information systems it is desirable to deal with single photons synchronized to an external clock, namely, triggerable single photons. Polarization states of single photons are also important as they enable polarization-qubit encoding of information.

The critical issue in producing single photons by a method other than by trivial attenuation of a beam is the very low concentration of photon emitter dispersed in a host, such that within a laser focal spot only one emitter becomes excited which can emit only one photon at a time. Most current SPSs, e.g., based on semiconductor heterostructures [15]-[19], [56], [57], operate only at liquid He temperature-a major impediment to widespread use. Of the known room-temperature (RT) SPSs, only those based on single-dye-molecule fluorescence [20]-[24] can be used in much higher speed systems than other RTSPSs. Alternatives such as color centers in diamond [25]-[28], [58] and colloidal semiconductor CdSe-ZnS quantum dots [29]-[31] possess unacceptably long fluorescence lifetimes. For instance, the diamond color center has a 11.6-ns [25] and 22.7 ns [27], [58] fluorescence life time in mono- and polycrystal, and CdSe-ZnS quantum dots one of $\sim 22$ ns [30].

The key advantage of dye molecules is that their excited-state life-time of only a few nanoseconds [32] permits excitation repetition rates above $\sim 100 \mathrm{MHz}$. In dye-based SPSs, one of the current challenges is dye bleaching. However, recently single terrylene molecules have been doped into p-terphenyl molecular crystals $\left(10^{-11}\right.$ moles of terrylene per mole of p-terphenyl) prepared by a sublimation procedure that produced tiny platelets [21], [22], [32]-[36]. In this host, the dye is protected from exposure to diffusing quenchers (such as oxygen), and benefits from strong phonon emission into the host, preventing rapid thermal decomposition of the dye under intense illumination. In [22], it was found that for "thick" p-terphenyl crystals $(\sim 10 \mu \mathrm{m})$, this system becomes extremely photostable, allowing hours of continuous illumination of individual molecules without photobleaching. It assures long-term spectral stability and repro- 
ducibility from one terrylene absorber to the next [22], [33], [34]. Pumped by periodic, short-pulse laser radiation, single photons were generated at predetermined times at pump-pulse-repetition rates within the accuracy of the emission lifetime ( $2.8 \mathrm{~ns})$. Technical implementation of this system is difficult as these monoclinic, sublimation-produced microcrystals are stress sensitive and fragile. In addition, terrylene's molecular dipole moment in the p-terphenyl host crystal takes on an orientation perpendicular to the platelet's surface (i.e., perpendicular to the incident light's E-field) [22]. This, in turn, leads to poor coupling with the polarized excitation light, prompting poor fluorescence emission even at high excitation intensities (saturation intensity is about $1 \mathrm{MW} / \mathrm{cm}^{2}$ at room temperature). In spite of the elegance of the terrylene/p-terphenyl experiments, this technology must be considered unrealistic for practical application. Its weak point is also a background from "ordinary photons" from out-of-focus molecules or Raman scattering, because of the very high pumping intensities required. Emitted photons are not polarized deterministically (there is no known, efficient method for aligning rapidly a multitude of micrometer-sized, monoclinic crystallites relative to one another). Note that noncrystalline, amorphous hosts, e.g., polymers [23], [24], do not 1) offer the same spectral stability in single-molecule emission even in the case of terrylene, 2) provide long-time protection against bleaching. To date, no crystal hosts other than the fragile, sublimated p-terphenyl flakes have been proposed in single-dye-molecule room temperature experiments.

This paper describes some new approaches toward an implementation of an efficient, deterministically polarized SPS on demand: 1) using liquid-crystal hosts (including liquid-crystal polymers) to preferentially align the emitter molecules for maximum excitation efficiency. Deterministic molecular alignment will also provide deterministically polarized output photons; 2) using planar-aligned cholesteric liquid crystal hosts [37] as one-dimensional (1-D) photonic-band-gap microcavities [38], [39] tunable to the dye fluorescence band; 3) using liquid crystal technology to prevent dye bleaching.

\section{Cholesteric Liquid CRystal 1-D-Photonic BAND-GAP MATERIALS}

In planar cholesterics (chiral nematics), that for visualization purposes can be described as consisting of a layered structure, the axes of the molecular director (rightmost set of arrows in Fig. 1) rotate monotonically to form a periodic helical structure with pitch $\mathrm{P}_{\mathrm{o}}$ [37]. With few exceptions, liquid-crystal media are nonchiral and require additives to induce the chiral order. Dependent on the chirality-inducing additive, the final structure may show either a right- or a left-handed sense of rotation.

When a solid, planar-aligned cholesteric is flipped on its side and inspected by a high-resolution tool such as an atomic-force-microscope, the periodic pitch becomes observable through height variations along the helical axis. For instance, Fig. 2 shows such a topography for a Wacker cyclo-tetrasiloxane-oligomer cholesteric liquid-crystal (OCLC) [40] platelet. It was cut from a planar-aligned OCLC coating on the substrate. Periodic stripes in the image correspond to one half of the pitch length.

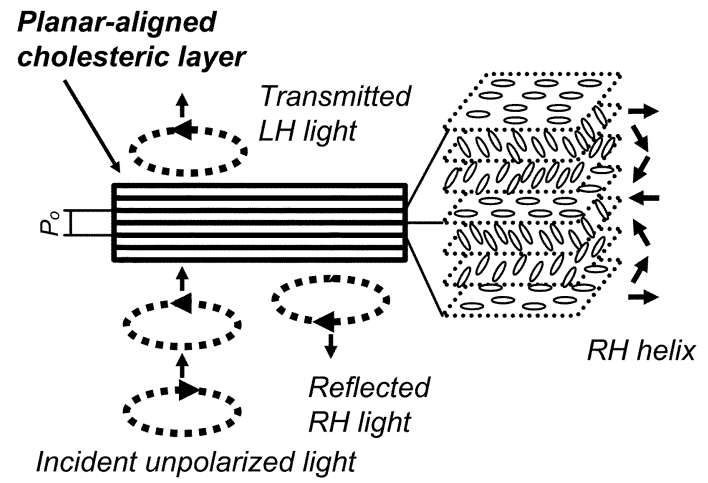

Fig. 1. Transmission and reflection by a cholesteric liquid crystal layer near selective reflection conditions.

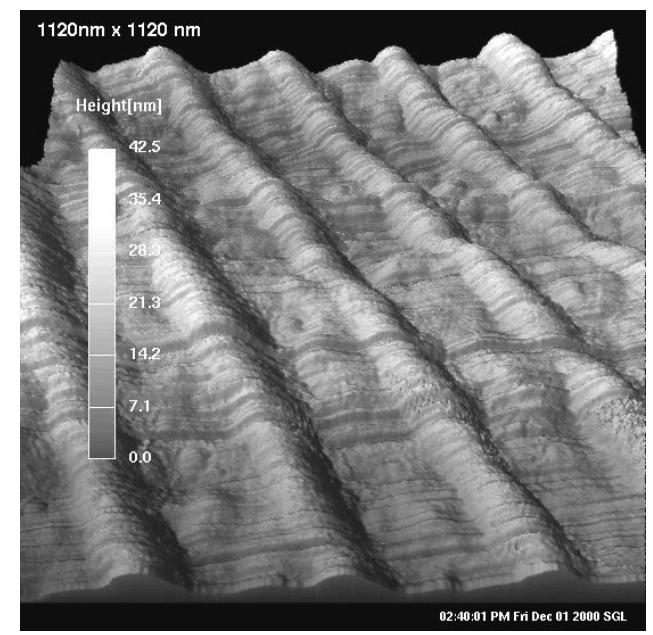

Fig. 2. Perspective view of the AFM-topographical image of a planar-aligned Wacker OCLC platelet $(1.12 \mu \mathrm{m} \times 1.12 \mu \mathrm{m}$ scan $)$.

For liquid-crystal thicknesses $\geq 10 \mu \mathrm{m}$, the reflectance of normally incident, circularly polarized light with electric-field vector-rotation opposite to the rotation of molecules in the helical structure (Bragg condition), approaches $100 \%$ within a band centered at $\lambda_{\mathrm{o}}=\mathrm{n}_{\mathrm{av}} \mathrm{P}_{\mathrm{o}}$ where $\mathrm{n}_{\mathrm{av}}=\left(\mathrm{n}_{\mathrm{e}}+\mathrm{n}_{\mathrm{o}}\right) / 2$ is the average of the ordinary and extraordinary refractive indices of the medium. This is the so-called selective reflection of cholesteric liquid crystals. The bandwidth is $\Delta \lambda=\lambda_{\mathrm{o}} \Delta \mathrm{n} / \mathrm{n}_{\mathrm{av}}$, where $\Delta \mathrm{n}=\mathrm{n}_{\mathrm{e}}-\mathrm{n}_{\mathrm{o}}$. Such a periodic structure can also be viewed as a 1-D photonic crystal, with a bandgap within which propagation of light is forbidden. For emitters located within such a structure, the rate of spontaneous emission is suppressed within the spectral stop band and enhanced near the band edge [41]. Several groups have reported lasing in photonic band-gap material hosts, including cholesteric liquid crystals [41]-[43], with spectral emission features underscoring the validity of this concept. Generation of strongly circularly polarized photoluminescence from planar-aligned cholesteric liquid crystals was also reported [44]-[46].

In our experiments, Wacker OCLC (see Fig. 3) was doped with terrylene (Fig. 4) at extremely low concentration such that the final sample contained only a few molecules per $\mu \mathrm{m}^{2}$ irradiation area. Cast from solution on single microscope-coverglass slips, samples ranged in thickness from $\sim 50$ $\mathrm{nm}$ to several micrometers. In some experiments, we also 


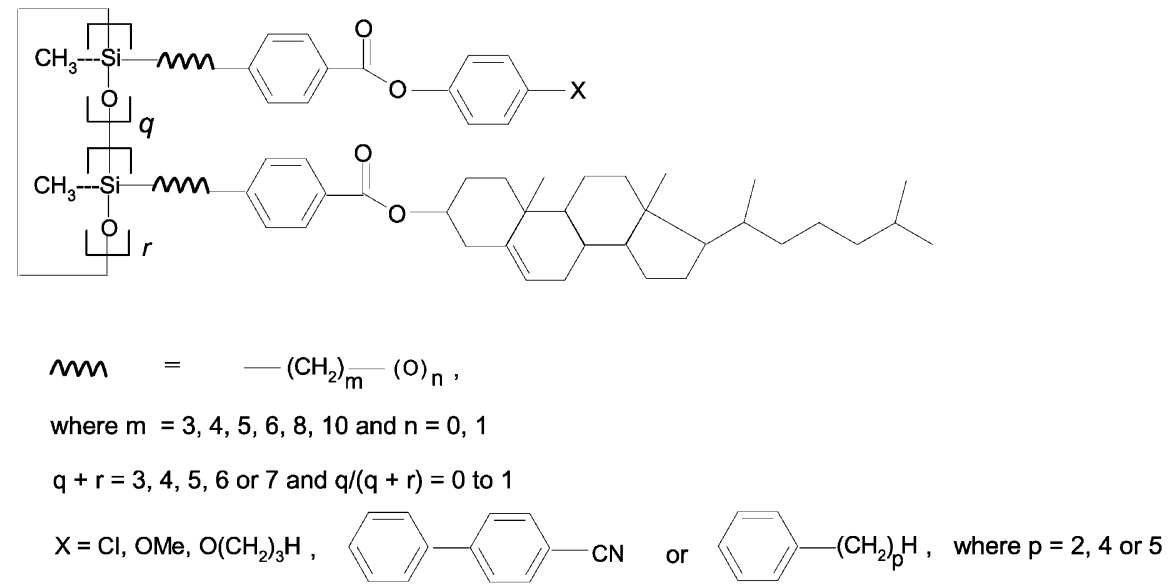

Fig. 3. Molecular structure of Wacker siloxane OCLC (from [40]).

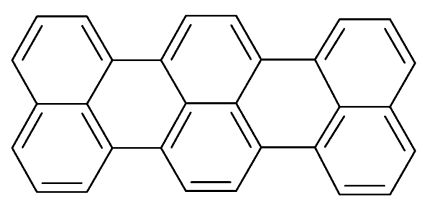

Fig. 4. Molecular structure of terrylene dye.

used terrylene-doped layers of monomeric, cyanobiphenyl liquid crystal 5CB with chiral additive CB15. The 5CB liquid crystal layers were placed between two microscope cover glass slips separated by glass-bead spacers. In order to minimize false fluorescence contributions by contaminants during single-molecule-fluorescence microscopy, rigorous cleaning of glass substrates is mandatory. For this purpose, the microscope glass slips (Corning, $0.17 \mathrm{~mm}$ thickness) were etched in piranhas solution [47] and rinsed in deionized water. Proper terrylene concentration for single-molecule fluorescence microscopy was established by iterative trial and error. In sequential dilution steps of terrylene in chlorobenzene solvent, solutions were spun onto glass slips and for each concentration confocal fluorescence microscopy determined the final emitter concentration per irradiation volume. Once single molecules were predominantly observed, the dilution endpoint was reached. This final terrylene solution was mixed with Wacker OCLC starting material (8\% weight concentration of oligomer). For planar alignment, standard buffing procedures could not be employed at the risk of introducing dirt particles. Two alternate methods were found satisfactory: either the film was flow aligned by letting the OCLC solution run down a vertically inclined glass slip, or a special glass-cylinder was rolled unidirectionally across a spin-coated OCLC heated to the liquid state $\left(\sim 100^{\circ} \mathrm{C}\right)$ and slowly cooled afterwards.

\section{ExPerimental Setup for Single-Dye Molecule FLUORESCENCE MICROSCOPY AND ANTIBUNCHING CORRELATION MEASUREMENTS}

Photon antibunching correlation measurements are carried out using a setup shown in Fig. 5. The terrylene-doped liquid

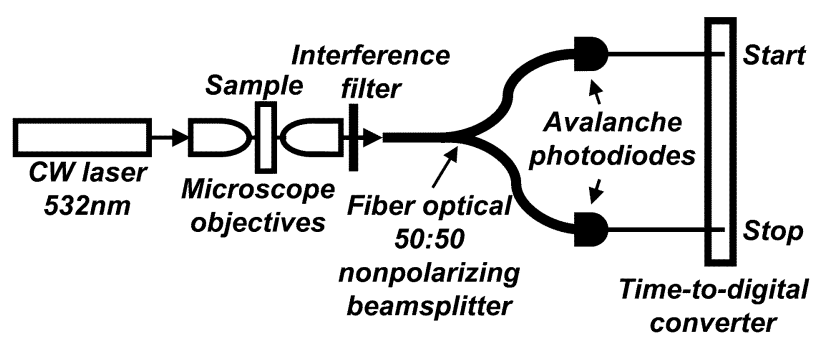

Fig. 5. Experimental setup for photon antibunching correlation measurements.

crystal sample is placed in the focal plane of a 0.8 N.A. microscope objective (Witec alpha-SNOM platform). The sample is attached to a piezoelectric, XYZ translation stage. Light emitted by the sample is collected by a confocal setup using a 1.25 N.A., oil-immersion objective together with an aperture in form of the $50-\mu \mathrm{m}$-core optical fiber. The $\mathrm{cw}$, spatially filtered through a single-mode fiber, linearly polarized (contrast $10^{5}: 1$ ), 532-nm diode-pumped Nd:YAG laser output excites single molecules. In focus, the intensities used are of the order of several $\mathrm{kW} / \mathrm{cm}^{2}$. The collection fiber is part of a nonpolarization-sensitive 50:50 fiber splitter that forms the two arms of a Hanbury Brown and Twiss correlation setup [48] (Fig. 5). Residual, transmitted excitation light is removed by two, additively placed, dielectric interference filters yielding a combined rejection of better than six orders of magnitude at $532 \mathrm{~nm}$.

Photons in the two Hanbury Brown and Twiss arms are detected by identical, cooled avalanche photodiodes in single-photon-counting Geiger mode (SPCM-AQR-14-FC, Perkin Elmer Optoelectronics, Vaudreuil, Canada). The time interval between two consecutively detected photons in separate arms is measured by a 68-ns-full-scale time-to-digital convertor (Model 7186, Phillips Scientific) using a conventional start-stop protocol. Within this convertor's linear range, the time uncertainty in each channel corresponds to $25 \mathrm{ps}$.

It was proved experimentally (see, e.g., [8], [20], [23]) that a very good approximation of the autocorrelation function $\mathrm{g}^{(2)}(\tau)$ comes directly from the coincidence counts (event distribution) $\mathrm{n}(\tau)$, for relatively low detection efficiency and therefore low 

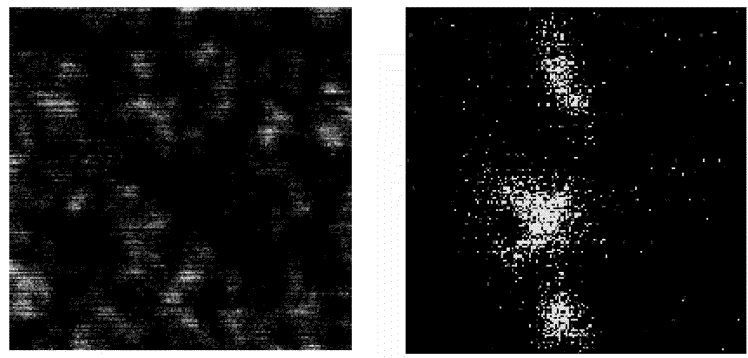

Fig. 6. Terrylene molecule fluorescence: left—single molecule fluorescence from the OCLC host; right-fluorescent-molecule clusters on a bare surface.

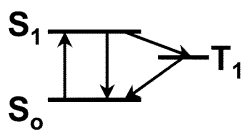

Fig. 7. Three-level model for molecular fluorescence.

counting rate. This justifies our assumption that $\mathrm{n}(\tau)$ is proportional to the autocorrelation function $\mathrm{g}^{(2)}(\tau)$. For single photons, $g^{(2)}(0)=0$ indicating the absence of pairs, i.e., antibunching.

\section{EXPERIMENTAL Results: Single-Dye Molecule}

FLUORESCENCE IN A CHOLESTERIC LIQUID CRYSTAL HOST.

Photon ANTIBUnChING CORRELATION MEASUREMENTS

Fig. 6 shows terrylene-dye-molecule-fluorescence images obtained by confocal fluorescence microscopy: left-single terrylene molecules embedded in a Wacker OCLC host; right-clusters of terrylene molecules spin coated from chlorobenzene solution onto a bare glass cover slip. For both images, the scan direction is from left to right and line by line from top to bottom. The scan dimensions are $10 \mu \mathrm{m} \times 10 \mu \mathrm{m}$. Most single molecules in these samples exhibited fluorescence blinking in time, with a period ranging from several milliseconds up to several seconds. In Fig. 6, this "blinking" behavior by single molecules manifests itself as bright and dark horizontal stripes in the image. These features are absent in emission images from clusters. Blinking is a common phenomenon and convincing evidence of the single-photon nature of the source. Several mechanisms are suggested for the explanation of blinking behavior, for instance, "shelving" (triplet blinking) to the long-living state, and fluctuations in the photo-physical parameters of the molecule and its local environment [49].

By modeling the molecule as a three-level system (singlet ground state $S_{o}$, excited state level $S_{1}$, and triplet state $T_{1}$ ) as depicted in Fig. 7, triplet blinking can be explained by a population buildup at the $T_{1}$ level that is often a dark state in fluorescence dye. With a single-pixel dwell time of $\sim 6 \mathrm{~ms}$ (Fig. 6) we are not able to resolve the lifetime of this triplet state $\left(\sim 10^{-4}\right.$ $\mathrm{s}$ for terrylene in a p-terphenyl [21]). The second mechanism is more plausible for the explanation of long-time intensity fluctuations although the details are not clearly understood. (See also discussion in [47] with some other suggestions).

Fig. 8 shows a coincidence-count histogram $\mathrm{n}(\tau)$ from host-free single terrylene molecules (left) and an assembly of

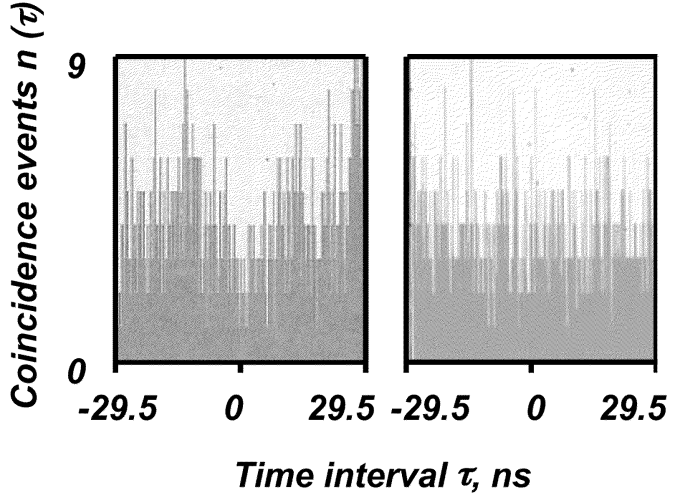

Fig. 8. The histograms of coincidence events of single-terrylene-molecule fluorescence on a bare glass substrate (left) and from an assembly of several uncorrelated molecules within the excitation volume (right).

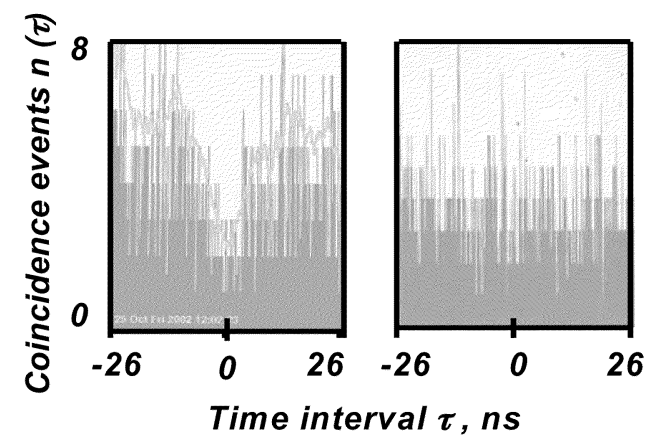

Fig. 9. The histograms of coincidence events of the single-terrylene-molecule-fluorescence on a bare glass substrate (left) and of the radiation of scattered light from the excitation laser beam (right).

many uncorrelated molecules within the excitation volume (Fig. 8, right).

The scan speed is $\sim 3 \mathrm{~s}$ per line (512 pixels). The left histogram exhibits a dip at $\tau=0$. The measured signal-to-background ratio of our experiments ranges from 2 to 30 , so the probability that a photon from the background triggers a coincidence with a photon from the molecule is very low. Because $n(\tau)$ is proportional to the autocorrelation function $\mathrm{g}^{(2)}(\tau), \mathrm{n}(0) \sim 0$ means that $g^{(2)}(0) \sim 0$ in our experiments. Two fluorescence photons are not observed within an arbitrarily short time interval. This fluorescence antibunching is due to the finite radiative lifetime of the molecular dipole and is therefore clear proof that we observed the emission from one and only one molecule. The histogram on the right from multiple, uncorrelated molecules shows no such dip at $\tau=0$, i.e., no antibunching.

To eliminate any potential for leaked excitation light causing the dip at $\tau=0$, the sample was replaced with a bare glass slide and one blocking interference filter was removed. The coincidence histogram for this condition is depicted in Fig. 9, right. No antibunching is observed.

Fig. 10 shows the results of doping terrylene into liquid crystals. The histogram of coincidence events $\mathrm{n}(\tau)$ (left) exhibits a dip at $\tau=0$, indicating photon antibunching in the fluorescence of the single molecules in the Wacker OCLC host; no antibunching is observed in the fluorescence from an assembly of several uncorrelated molecules in the same host, different sample (right histogram). The left histogram 


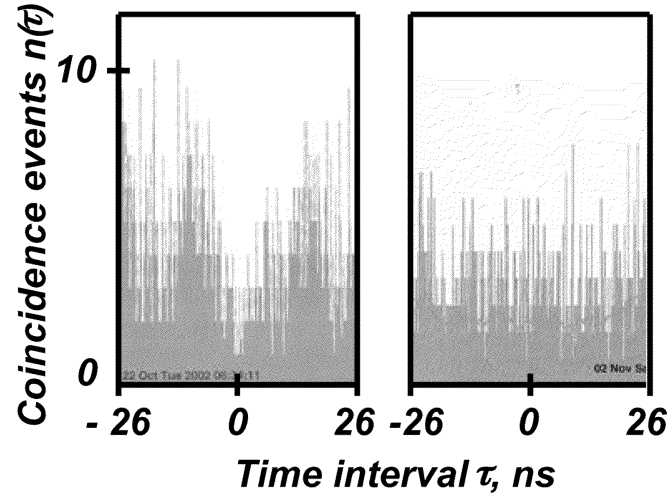

Fig. 10. Histograms of coincidence events of the single-terrylene-molecule-fluorescence in a Wacker OCLC host (left) and of an assembly of several uncorrelated molecules (right).

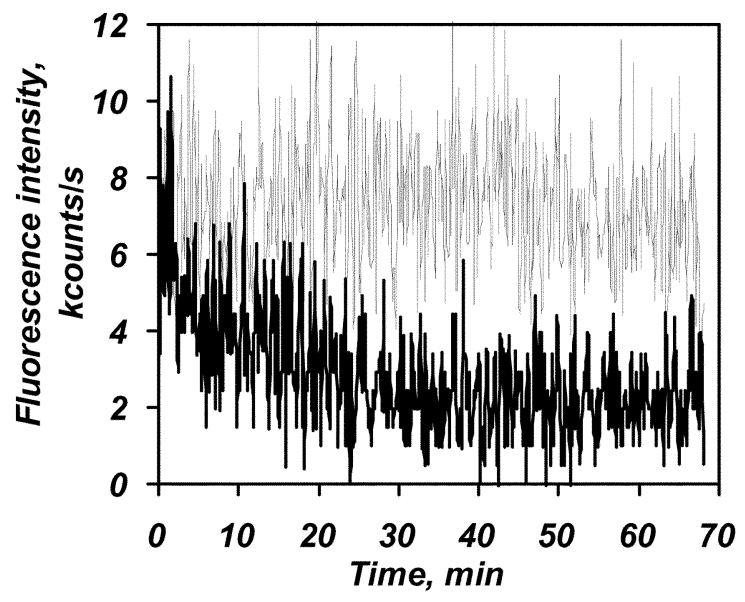

Fig. 11. Fluorescence bleaching behavior of an assembly of terrylene molecules as a function of time and in two different liquid-crystal hosts.

in Fig. 10 is noteworthy in that it demonstrates that several single molecules can sequentially contribute to an antibunching histogram without loss of $\tau=0$ contrast, as in practice the long integration time and competing molecule-bleaching events make obtaining an entire, good-contrast histogram from only one molecule too much a matter of luck. When the initial single molecule was bleached, the sample was advanced to another single molecule while the photon-correlation count continued. This finding is crucial for future device implementation.

\section{EXPerimental Results: PreVEnting Dye Bleaching IN LIQUID CRYSTAL HOSTS}

Practical device implementation also depends on photochemical stability of both emitters and hosts. We increased terrylene fluorescence stability in monomeric liquid crystal hosts by saturating, prior to cell assembly, the liquid crystals with helium in a sealed glove-box for one hour. Oxygen that is mostly responsible for dye bleaching is displaced by helium during this procedure. Fig. 11 shows fluorescence-bleaching results of terrylene molecules at two-orders-of-magnitude higher concentration than in single-molecule experiments in different liquid crystal hosts: either immobilized in an oligomer cholesteric liquid crystal or dissolved in monomeric cyanobiphenyl 5CB saturated with helium (both at identical excitation intensity and identical terrylene volume concentration). Over the course of more than one hour, no dye bleaching was observed in the oxygen-depleted liquid crystal host (upper curve). Dye bleaching was avoided also in [50] for $\sim 1$ hour of irradiation by placing the sample under nitrogen.

Dye bleaching is not a critical impairment for an efficient SPS, but it is an important factor for device simplicity and cost. When one molecule is bleached the system can be rapidly realigned to utilize another isolated dye molecule, allowing practically continuous source action (see left histograms on Figs. 8-10).

\section{CONCLUSION}

A robust room temperature SPS based on fluorescence from a single-dye-molecule (fluorescence antibunching) was demonstrated for the first time for liquid crystal hosts. Planar-aligned, 1-D photonic-band-gap structures in dye-doped cholesteric oligomers were prepared. Avoiding bleaching of the terrylene dye molecules for excitation times $>1 \mathrm{~h}$ was achieved by innovative preparation procedures.

Estimating the efficiency of our SPS using conservative value of parameters, we compare the number of exciting 532-nm photons/s $\mathrm{N}_{\text {inc }}$ incident on the absorption cross-section area $\sigma$ of a terrylene molecule with a measured photon counting rate from a single molecule $\mathrm{N}_{\text {out }}=3 \mathrm{kc} / \mathrm{s}$. For the laser power incident on the sample $\sim 17.5 \mu \mathrm{W}$, a beam radius $\sim 0.25 \mu \mathrm{m}$, and using measured value of $\sigma \sim 5 \times 10^{-17} \mathrm{~cm}^{-2}$ for terrylene molecules [21], [23], we arrive at $\mathrm{N}_{\mathrm{inc}}=1.2 \times 10^{6}$ photons $/ \mathrm{s}-\mathrm{mol}$.

We can evaluate a probability $\mathrm{p}_{\alpha}$ for a single photon to be emitted into an optical fiber core of our Hanbury Brown-Twiss setup (Fig. 5), from the following expression: $2 \mathrm{~N}_{\text {out }}=0.95 \mathrm{~N}_{\text {inc }} \mathrm{p}_{\alpha} \mathrm{DQ}$. Here $\mathrm{D}=0.2$ is the measured coupling efficiency of the fiber optics used in this setup, $\mathrm{Q}=0.64$ is the photon detection efficiency of the avalanche photodiode (APD) at $579 \mathrm{~nm}, 0.95$ is the coupling efficiency from the fiber to the APD-FC-connector. From this data we deduce that $\mathrm{p}_{\alpha} \approx 4 \%$. This rather surprisingly large value is strongly dependent on the measured absorption cross section for which we used a conservative estimate [21].

Our current SPS performance with linear polarized light does not yet use all the capabilities offered by the liquid crystal host to increase the excitation and collection efficiency. We can estimate how the probability $\mathrm{p}_{\alpha}$ might be improved. Using the experimental data of [51] on the dependence of the fluorescence intensity on the incident polarization of dye molecule in a p-terphenyl host, the count rate may be increased at least by $2.6-4.3$ times by the alignment of the liquid crystal/dye molecules relative to the incident polarization. For lack of literature data about the extraction efficiency for an emitter in a planar-cholesteric liquid crystal distributed feedback cavity, we will use the experimental data on a small-number dye molecule fluorescence in a planar microcavity [8], [52]. These results show that the efficiency improves a factor between two and three with the microcavity compared to the dye molecules without the cavity. It is safe to say that an SPS efficiency increase of up to one order of magnitude can be expected using planar alignment of a 
cholesteric host whose photonic band-gap matches the dye fluorescence band.

The probability of two-photon emission $\mathrm{P}_{2}$ is approximately $\mathrm{P}_{2}=\mathrm{C}_{\mathrm{N}}(0) \mathrm{P}_{1}^{2} / 2$ if $\mathrm{P}_{2}$ is much smaller than unity [27], [58]. $\mathrm{P}_{1}$ is the probability for single-photon emission, $\mathrm{C}_{\mathrm{N}}(0)$ is the zero time normalized coincidence rate that can be taken directly as the correlation function $\mathrm{g}^{(2)}(0)$. For Poissonian light $\mathrm{C}_{\mathrm{N}}(0)=1$. In our case, for single terrylene molecule fluorescence in a Wacker oligomer liquid crystal host (Fig. 10) $\mathrm{C}_{\mathrm{N}}(0)=0.25-0.33$. It means that the rate of two-photon pulses is three-four times lower than for Poissonian light. It should be noted that a probability $\mathrm{p}_{\alpha}$ introduced earlier, $\mathrm{p}_{\alpha}=$ $\alpha \mathrm{P}_{1}$. Here $\alpha$ is a collection efficiency.

The authors' future work is directed toward increasing the efficiency, life, and polarization purity of the SPS by improved selection of dye, liquid crystal, and the photonic-band-gap structure matching with the dye fluorescence band. A pulsed-laser source will be used to create a real quantum cryptography system with a cholesteric liquid crystal SPS on demand.

\section{ACKNOWLEDGMENT}

The OCLC starting material from Dr. F. Kreuzer of Wacker, Munich, is gratefully acknowledged. The authors thank L. Novotny, K. Marshall, J. Shojaie, T. Kosc, and O. Hollricher for advice and help, S. Papernov for help with AFM-imaging of OCLC, and J. Starowitz for support in the optical materials laboratory.

\section{REFERENCES}

[1] M. A. Nielsen and I. L. Chuang, Quantum Computation and Quantum Information. Cambridge, U.K.: Cambridge Univ. Press, 2001.

[2] D. Bouwmeester, A. Ekert, and A. Zeilinger, Eds., The Physics of Quantum Information: Quantum Cryptography, Quantum Teleportation, Quantum Computation. Berlin, Germany: Springer, 2000.

[3] P. G. Kwiat, "Focus on quantum cryptography," New J. Phys., no. 4, 2002.

[4] N. Gisin, G. Ribordy, W. Tittel, and H. Zbinden, "Quantum cryptography," Rev. Mod. Phys., vol. 74, pp. 145-195, 2002.

[5] Technologies for quantum communications, in J. Modern Opt., J. G. Rarity, N. Gisin, and G. S. Buller, Eds., vol. 48, no. 13, 2001.

[6] Experimental proposals for quantum computation, in Fortschritte der Physik, S. L. Braunstein and H.-K. Lo, Eds., vol. 48, no. 9, pp. 767-1138, 2000.

[7] Solid State Sources for Single Photons, S4P Consort. Eur. Commun., Project IST-1999-10243 (2000-2001). Available: www.iota.u-psud.fr/ S4P/. [Online]

[8] P. Jonsson, "Generation, detection and applications of single photons," Doctoral dissertation, Royal Inst. of Technol., Stockholm, 2002.

[9] D. F. Walls and G. J. Milburn, Quantum Optics. Berlin-New York: Springer, 1995, p. 42.

[10] U. Mets, "Antibunching and rotational diffusion in FCS," in Fluorescence Correlation Spectroscopy. Theory and Applications, R. Rigler and E. S. Elson, Eds. $\quad$ Berlin-New York: Springer, 2001, pp. 346-359.

[11] E. Klarreich, "Can you keep a secret?," Nature, vol. 418, pp. 270-272, 2002.

[12] E. Knill, R. Laflamme, and G. J. Milburn, "A scheme for efficient quantum computation with linear optics," Nature, vol. 409, pp. 46-52, 2001.

[13] J. Calsamiglia, "Quantum information processing and its linear optical implementation," Academic dissertation, Univ. Helsinki, Finland, 2001

[14] T. B. Pittman, B. C. Jacobs, and J. D. Franson, "Demonstration of nondeterministic quantum logic operation using linear optical elements," Phys. Rev. Lett., vol. 88, pp. 257 902-1-257 902-4, 2002.

[15] J. Kim, O. Benson, H. Kan, and Y. A. Yamamoto, "Single photon turnstile device," Nature, vol. 397, pp. 500-503, 1999.
[16] E. Moreau, I. Robert, J. M. Gérard, I. Abram, L. Manin, and V. ThieryMieg, "Single-mode solid-state single photon source based on isolated quantum dots in pillar microcavities," Appl. Phys. Lett., vol. 79, pp. 2865-2867, 2001.

[17] P. Michler, A. Kiraz, C. Becher, W. V. Schoenfeld, P. M. Petroff, L. Zhang, E. Hu, and A. Imamoglu, "A quantum dot single-photon turnstile device," Science, vol. 290, pp. 2282-2285, 2000.

[18] C. Santori, M. Pelton, G. Solomon, Y. Dale, and Y. Yamamoto, "Triggered single photons from a quantum dot," Phys. Rev. Lett., vol. 86, pp. 1502-1505, 2001

[19] Z. L. Yuan, B. E. Kardynal, R. M. Stevenson, A. J. Shields, C. J. Lobo, K. Cooper, N. S. Beattie, D. A. Ritchie, and M. Pepper, "Electrically driven single-photon source," Science, vol. 295, pp. 102-105, 2002.

[20] W. P. Ambrose, P. M. Goodwin, J. Enderlein, D. J. Semin, J. C. Martin, and R. A. Keller, "Fluorescence photon antibunching from single molecule on a surface," Chem. Phys. Lett., vol. 269, pp. 365-370, 1997.

[21] R. A. Fleury, J.-M. Segura, G. Zumofen, B. Hecht, and U. P. Wild, "Nonclassical photon statistics in single-molecule fluorescence at room temperature," Phys. Rev. Lett., vol. 84, pp. 1148-1151, 2000.

[22] B. Lounis and W. E. Moerner, "Single photons on demand from a single molecules at room temperature," Nature, vol. 407, pp. 491-493, 2000.

[23] F. Treussart, A. Clouqueur, C. Grossman, and J.-F. Roch, "Photon antibunching in the fluorescence of a single dye molecule embedded in a thin polymer film," Opt. Lett., vol. 26, pp. 1504-1506, 2001.

[24] F. Treussart, R. Alleaume, V. Le Floch, L. T. Xiao, J. M. Courty, and J. F. Roch, "Direct measurement of the photon statistics of a triggered single photon source," Phys. Rev. Lett., vol. 89, no. 9, pp. 093 601-093 604, 2002.

[25] C. Kurtsiefer, S. Mayer, P. Zarda, and H. Weinfurter, "Stable solid-state source of single photons," Phys. Rev. Lett., vol. 85, pp. 290-293, 2000.

[26] R. Brouri, A. Beveratos, J.-P. Poizat, and P. Grangier, "Photon antibunching in the fluorescence of individual color centers in diamond," Opt. Lett., vol. 25, pp. 1294-1296, 2000.

[27] A. Beveratos, R. Brouri, T. Gacoin, J.-P. Poizat, and P. Grangier, "Nonclassical radiation from diamond nanocrystals," Phys. Rev. A, vol. 64, p. 061 802(R), 2001.

[28] A. Beveratos, R. Brouri, T. Gacoin, A. Villing, J. P. Poizat, and P. Granger, "Single photon quantum cryptography," Phys. Rev. Lett., vol. 89, no. 18, pp. 187901-187904, 2002.

[29] P. Michler, A. Imamoglu, M. D. Mason, P. J. Carson, G. F. Strouse, and S. K. Buratto, "Quantum correlation among photons from a single quantum dot at room temperature," Nature, vol. 406, pp. 968-970, 2000.

[30] B. Lounis, H. A. Bechtel, D. Gerion, P. Alivisatos, and W. E. Moerner, "Photon antibunching in single CdSe/ZnS quantum dot fluorescence," Chem. Phys. Lett., vol. 329, pp. 399-404, 2000.

[31] G. Messin, J. P. Hermier, E. Giacobino, P. Desbiolles, and M. Dahan, "Bunching and antibunching in the fluorescence of semiconductor nanocrystals," Opt. Lett., vol. 26, pp. 1891-1893, 2001.

[32] T.Th. Basché, W. E. Moerner, M. Orrit, and U. P. Wild, Eds., Single Molecule Optical Detection, Imaging and Spectroscopy. Weinheim, Germany: VCH, 1997.

[33] L. Fleury, B. Sick, G. Zumofen, B. Hecht, and U. P. Wild, "High photostability of single molecules in an organic crystal at room temperature observed by scanning confocal optical microscopy," Molec. Phys., vol. 95, pp. 1333-1338, 1998.

[34] F. Kulzer, F. Koberling, T.Th. Christ, A. Mews, and T.Th. Basché, “Terrylene in p-terphenyl: single-molecule experiment at room temperature," Chem. Phys., vol. 247, pp. 23-24, 1999.

[35] F. Kulzer, "Untersuchungen zum nichtphotochemischen lochbrennen auf einzelmolekülebene am modellsystem terrylen in p-Terphenyl," Ph.D. dissertation, Mainz, 2000.

[36] J. Michaelis, "Microscopie mit einem einzelnen molekül als lichtquelle," Ph.D. dissertation, Konstanz, 2000.

[37] S. Chandrasekhar, Liquid Crystals. Cambridge: Cambridge Univ. Press, 1977.

[38] E. Yablonovitch, "Inhibited spontaneous emission in solid-state physics and electronics," Phys. Rev. Lett., vol. 58, pp. 2059-2062, 1987.

[39] S. John, "Strong localization of photons in certain disordered dielectric superlattices," Phys. Rev. Lett., vol. 58, pp. 2486-2489, 1987.

[40] T. J. Bunning and F.-H. Kreuzer, "Cyclosiloxane-based liquid-crystalline materials,” Trends Polym. Sci., vol. 3, no. 10, pp. 318-323, 1995.

[41] V. P. Kopp, B. Fan, H. K. M. Vithana, and A. Z. Genack, "Low threshold lasing at the edge of a photonic stop band in cholesteric liquid crystals," Opt. Lett., vol. 23, pp. 1707-1709, 1998.

[42] I. P. Il'chishin, E. A. Tikhonov, V. G. Tishchenko, and M. T. Shpak, "Generation of a tunable radiation by impurity cholesteric liquid crystals," JETP Lett., vol. 32, pp. 24-27, 1980. 
[43] H. Finkelman, S. T. Kim, A. Munoz, and P. Palffy-Muhoray, "Tunable mirrorless lasing in cholesteric liquid crystalline elastomers," Adv. Mater, vol. 13, no. 14, pp. 1069-1072, 2001

[44] S. H. Chen, D. Katsis, A. W. Schmid, J. C. Mastrangelo, T. Tsutsui, and T. N. Blanton, "Circularly polarized light generated by photoexcitation of luminophores in glassy liquid-crystal films," Nature, vol. 397, pp. 506-508, 1999

[45] D. Katsis, A. W. Schmid, and S. H. Chen, "Mechanistic insight into circularly polarized photoluminescence from chiral-nematic film," Liq. Cryst., vol. 26, pp. 181-185, 1999.

[46] A. Y.A. Yu Bobrovsky, N. I. Boiko, V. P. Shibaev, and J. H. Wendorff, "Cholesteric mixtures with photochemically tunable, circularly polarized fluorescence," Adv. Mater, vol. 15, no. 3, pp. 282-287, 2003.

[47] F. Vargas, O. Hollricher, O. Marti, G. de Schaetzen, and G. Tarrach, "Influence of protective layers on the blinking of fluorescent singlemolecules observed by confocal microscopy and scanning near field optical microscopy," J. Chem. Phys., vol. 117, no. 2, pp. 866-871, 2002.

[48] R. Hanbury Brown and R. Q. Twiss, "Correlation between photons in two coherent beams of light," Nature, vol. 177, no. 4497, pp. 27-29, 1956.

[49] A. Molski, "Photon-counting distribution of fluorescence from a blinking molecule," Chem. Phys. Lett., vol. 324, pp. 301-306, 2000.

[50] L. A. Deschenes and D. A. Vanden Bout, "Single-molecule studies of heterogeneous dynamics in polymer melts near the glass transition," Science, vol. 292, pp. 255-258, 2001.

[51] M. Croci, H.-J. Müschenborn, and U. P. Wild, "Polarization and lifetime measurements, external perturbations and microscopy," in Single Molecule Optical Detection, Imaging and Spectroscopy, T.Th. Basché, W. E. Moerner, M. Orrit, and U. P. Wild, Eds. Weinheim: VCH, 1997, p. 73.

[52] S. C. Kitson, P. Jonsson, J. G. Rarity, and P. R. Tapster, "Intensity fluctuation spectroscopy of small numbers of dye molecules in a microcavity," Phys. Rev. A, vol. 58, pp. 620-627, 1998.

[53] Efficient Linear Optics Quantum Computation (2000, June 20). Available: http://xxx.lanl.gov/abs/quant-ph/0006088 [Online]

[54] T. B. Pittman, B. C. Jacobs, and J. D. Franson, "Probabilistic quantum logic operations using polarizing beam splitters," Phys. Rev. A, vol. 64, pp. 062311-1-062311-9, 2001.

[55] A. Shields, "Quantum logic with light, glass and mirrors," Science, vol. 297, pp. 1821-1822, 2002.

[56] A. Imamoglu and Y. Yamamoto, "Turnstile device for heralded single photons: coulomb blockade of electron and hole tunneling in quantum confined p-i-n heterojunctions," Phys. Rev. Lett., vol. 72, pp. 210-213, 1994.

[57] M. Pelton, C. Santori, J. Vučković, B. Zhang, G. S. Solomon, J. Plant, and Y. Yamamoto, "Efficient source of single photons: a single quantum dot in a micropost microcavity," Phys. Rev. Lett., vol. 89, p. 233602 , 2002.

[58] A. Beveratos, S. Kuhn, R. Brouri, T. Gacoin, J. P. Poizat, and P. Grangier, "Room temperature stable single-photon source," Eur. Phys. J. D, vol. 18, pp. 191-196, 2002.

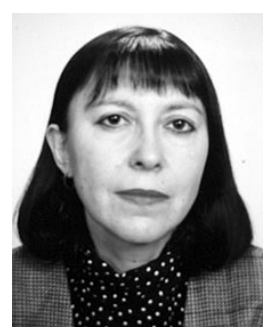

Svetlana G. Lukishova was born in Moscow, USSR She received the B.S., M.S., and Ph.D. degrees in physics and mathematics from the Moscow Institute of Physics and Technology (FizTech). Her Ph.D. dissertation in 1977 was conducted in the P.N. Lebedev Physical Institute, USSR Academy of Sciences. It involved the spatial and temporal profile formation of laser-thermonuclear fusion-system radiation and was supervised by Prof. P. P. Pashinin and Nobel prize winner Prof. A. M. Prokhorov.

She was a Research Staff Member of the Troitsk Branch of the I.V. Kurchatov Institute of Nuclear Power (TRINITI) from 1976 to 1981, and with the Institute of Radiongineering and Electronics of the USSR/Russian Academy of Sciences, Moscow, from 1981 to 1998, and Senior Scientist in 1987. From 1994 to 1997, she was a Senior Scientist with FizTech. In 1997 she was a Visiting Scientist with the Liquid Crystal Institute, Kent State University, Kent, OH. In 1999 she joined the Institute of Optics, University of Rochester, Rochester, NY, where she is a Senior Scientist. Her research interests include both optical radiation and material properties, in particular nonlinear optics of liquid crystals, single-molecule fluorescence microscopy, quantum optics, and laser physics.

Dr. Lukishova is a member of the Optical Society of America and the International Liquid Crystal Society.

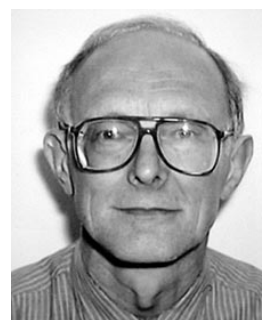

Ansgar W. Schmid was born in Salzburg, Austria. He received the B.S., M.S., and Ph.D. degrees from the Technical University of Vienna, Austria. His M.S. diploma was conducted in CERN, Switzerland, and the Ph.D. thesis research was performed at the Research Institute for Engineering Science at the Bendix Research Laboratories under an exchange program between Wayne State University, Detroit, MI, and the Technical University of Vienna. His Ph.D. research involved multiphoton-induced emission of halogen atoms from alkali halide crystals.

He was with the Physical Division of the National Research Council (Ottava), and was an Assistant Professor with the Washington State University,Pullman, and has been with the Laboratory for Laser Energetics, University of Rochester, Rochester, NY, for almost 20 years, where he is a Group Leader. His interests lie in devising new cost-effective materials and devices for use on high-peakpower lasers, specifically ICF drivers, manufacturing issues of organics in general, polymers and liquid crystals in particular, and laser-induced damage of materials. Most recently, these interests have focused on nanometrology with near-field, atomic force, and confocal microscopy.

Dr. Schmid is a member of the Optical Society of America.

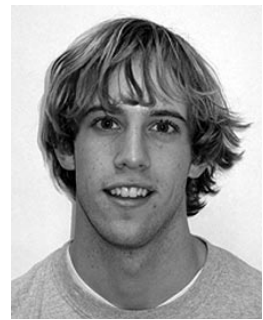

Andrew J. McNamara was born in Albany, NY.

$\mathrm{He}$ is currently a sophomore student with the Institute of Optics, University of Rochester, Rochester, NY. This is his first paper in a refereed scientific journal.

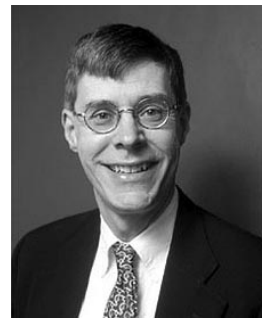

Robert W. Boyd was born in Buffalo, NY. He received the B.S. degree in physics from the Massachusetts Institute of Technology, Cambridge, and the $\mathrm{Ph} . \mathrm{D}$. degree in physics in 1977 from the University of California at Berkeley. His Ph.D. thesis was supervised by Prof. C. H. Townes and involved the use of nonlinear optical techniques in infrared detection for astronomy.

He joined the faculty of the Institute of Optics, University of Rochester, in 1977 and since 1987 has held the position of Professor of Optics. Since July 2001, he has also held the position of the M. Parker Givens Professor of Optics, and since July 2002, has also held the position of Professor of Physics. His research interests include studies of nonlinear optical interactions, studies of the nonlinear optical properties of materials, the development of photonic devices including photonic biosensors, and studies of the quantum statistical properties of nonlinear optical interactions. He has written two books, coedited two anthologies, published more than 200 research papers, and has been awarded five patents.

Prof. Boyd is a Fellow of the Optical Society of America and of the American Physical Society and is the past chair of the Division of Laser Science of the American Physical Society.

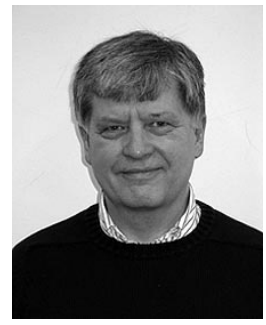

Carlos R. Stroud, Jr. was born in Owensboro, KY. He received the A.B. degree in physics and mathematics from Centre College of Kentucky, Danville, and the Ph.D. degree in physics from Washington University, St. Louis, MO, in 1969.

$\mathrm{He}$ has been a member of the faculty of the University of Rochester, Rochester, NY, since 1969 and currently holds appointments of Professor of Optics and Professor of Physics. He has worked in areas of theoretical and experimental quantum optics, notably in carrying out the first high-resolution CW dye-laser experiments in sodium, first observation of the three-peaked resonance fluorescence spectrum, observation of coherent population trapping in atoms, extensive studies of wave packet formation in Rydberg atomic states, and the interaction of terahertz radiation with matter.

Prof. Stroud is a Fellow of the Optical Society of America and of the American Physical Society, and currently serves as Distinguished Traveling Lecturer of the Division of Laser Science of the American Physical Society. 